\title{
Role of $n-3$ series polyunsaturated fatty acids in cardiovascular disease prevention
}

REVIEW

\author{
This article was published in the following Dove Press journal: \\ Nutrition and Dietary Supplements \\ I4 September 201 I \\ Number of times this article has been viewed
}

\author{
Andy H Lee' \\ Naoko Hiramatsu ${ }^{2}$ \\ 'School of Public Health, Curtin \\ Health Innovation Research Institute, \\ Curtin University, Perth,WA, \\ Australia; ${ }^{2}$ Laboratory of Nutritional \\ Science, School of Human Science and \\ Environment, University of Hyogo, \\ Himeji, Hyogo, Japan
}

\begin{abstract}
Cardiovascular disease is a major cause of morbidity and mortality worldwide. Its prevention through a healthy lifestyle and appropriate diet is important. Omega-3 polyunsaturated fatty acids (n-3 PUFA) therapy has shown promise in both primary and secondary prevention of cardiovascular disease. This commentary discusses the nutritional role of $n-3$ PUFA, including its metabolism and physiological role, comparison with n- 6 series PUFA, as well as complications due to deficiency. Clinical use of n-3 PUFA for the prevention and treatment of cardiovascular disease, recommended intake, and potential adverse effects will also be examined. The available scientific evidence suggests that its supplementation and clinical use ranging from 0.4 to $1 \mathrm{~g}$ /day can provide tangible benefits. However, further studies are required to determine optimal dosing and the relative ratio of docosahexaenoic acid and eicosapentaenoic acid that provides maximal cardioprotection and treatment of cardiovascular disease.
\end{abstract}

Keywords: alpha-linolenic acid, docosahexaenoic acid, eicosapentaenoic acid, cardiovascular disease, fish oil, polyunsaturated fatty acids

\section{Introduction \\ Cardiovascular diseases}

Cardiovascular diseases are a group of disorders of the heart and blood vessels, and include coronary heart disease, cerebrovascular disease (stroke), raised blood pressure (hypertension), peripheral artery disease, rheumatic heart disease, congenital heart disease, and heart failure. According to the World Health Organization, cardiovascular diseases are the number one cause of death globally and claim 17 million lives each year. ${ }^{1}$ By 2030, almost 24 million people will die from cardiovascular disease, mainly from heart disease and stroke. These are projected to remain the single leading causes of death. ${ }^{1}$ In the US, cardiovascular diseases account for more than one-third (34.3\%) of deaths annually, and are responsible for nearly three million Americans reporting disability. The costs of cardiovascular disease are also staggering. In 2010, the total cost, including health care services, medications, and lost productivity, was estimated to be over $\$ 503$ billion in the US. ${ }^{2}$ Similarly, the National Heart Foundation of Australia reported that cardiovascular diseases are the leading cause of mortality and morbidity in Australia, killing one person nearly every ten minutes. ${ }^{3}$ Despite improvements over the last few decades, cardiovascular diseases remain the second largest disease burden to our society after cancers. As the population ages, the economic impact of cardiovascular diseases on the health care system will become even greater. Tobacco smoking, an unhealthy diet, physical inactivity, and high alcohol consumption increase the risk of cardiovascular disease. Indeed, behavioral and dietary risk factors
Correspondence: Andy H Lee

School of Public Health,

Curtin University, GPO Box U 1987 ,

Perth, WA, 6845, Australia

$\mathrm{Tel}+61892664180$

Fax +61892662958

Email andy.lee@curtin.edu.au 
are responsible for about $80 \%$ of coronary heart disease and cerebrovascular disease. ${ }^{1}$

Interestingly, both the incidence and mortality rate for cardiovascular diseases are much lower in Japan than other countries, ${ }^{4}$ which may be attributed to the high consumption of fish and seafood by the Japanese population. ${ }^{5}$ The fact that fish is abundant in omega-3 polyunsaturated fatty acids has opened an effective venue for the prevention and treatment of this disease by either dietary modifications or pharmacological supplementation.

\section{Polyunsaturated fatty acids}

Fat is one of the energy sources, and is indispensable for physiological functions, such as delivery of lipid-soluble vitamins, materials of biomembranes, and bioactive substances. Triglycerides are the most common fat in our diet. The term "fat" refers to triacylglycerols, which are composed of three fatty acid molecules attached to a glycerol backbone. Fatty acids consist of a hydrocarbon chain and a carboxyl group. Depending on the structural difference, they are categorized into two major groups, saturated fatty acids and unsaturated fatty acids, with or without one or more double bonds. Saturated fatty acids, the substantial components in animal fat, have only single bonds in the hydrocarbon chain. Unsaturated fatty acids have one or more double bonds in the hydrocarbon chain. Furthermore, the unsaturated fatty acids are classified into two groups, monounsaturated fatty acids and polyunsaturated fatty acids (PUFA).

There are two main types of bioactive PUFA, namely, the omega-6 (n-6) series, and the omega-3 (n-3) series. The n-6 series comprises linoleic acid (18:2 n-6), gammalinolenic acid (18:3 n-6), arachidonic acid (20:4 n-6), and others. The $n-3$ series includes alpha-linolenic acid (18:3 n-3), eicosapentaenoic acid (20:5 n-3), docosapentaenoic acid (22:5 n-3), and docosahexaenoic acid (22:6n-3). Both n-3 PUFA and n-6 PUFA are important constituents of all cell membranes. Because PUFA such as linoleic acid and alpha-linolenic acid cannot be synthesized in the human body, that is, they are unable to desaturate $\mathrm{C}-\mathrm{C}$ bonds of the $n-3$ and $n-6$ carbons involved in the hydrocarbon chain of fatty acids, these PUFA must be obtained from the diet and thus are called essential fatty acids. Common sources of n-3 PUFA include fish oils (eicosapentaenoic acid and docosahexaenoic acid) and some plant oils such as canola and flaxseed oils (alpha-linolenic acid). While an overall reduction of fat intake is advisable especially in Western countries, more emphasis has been placed on the relative proportion of individual fatty acids.

\section{Scope of study}

In the literature, observational and experimental studies, especially clinical trials, have found n-3 PUFA to be important in the protection against cardiovascular disease. Evidence has suggested cardiovascular benefits for patients following myocardial infarction and in those with heart failure. Besides, the level of n-3 PUFA may have both prognostic and diagnostic utility in cardiovascular disease risk assessment.

In this commentary, we consider the scientific evidence showing the potential benefits of n-3 PUFA, particularly eicosapentaenoic acid and docosahexaenoic acid, in primary and secondary cardiovascular disease prevention. Our review will include the metabolism of n-3 PUFA, its physiological role, a comparison with n-6 PUFA, as well as complications due to deficiency. Potential clinical use for cardiovascular disease risk management will then be examined, including the recommended dosage that can provide maximal cardioprotection. However, detailed physiology and biological mechanisms underlying the benefits of the n-3 PUFA series are beyond the scope of this commentary.

\section{Metabolism and physiological role of PUFA Metabolism}

Humans can in theory synthesize longer n-6 and n-3 FA from the essential fatty acids, linoleic acid and alpha-linolenic acid, respectively, through a series of desaturation (addition of a double bond) and elongation (addition of two carbon atoms) reactions. However, these activities are known to be weak, and the human body can only convert small amounts of alpha-linolenic acid into eicosapentaenoic acid (about $5 \%)$ and docosahexaenoic acid $(<0.5 \%){ }^{6}$ The n-6 PUFA and n-3 PUFA are metabolized through different metabolic pathways to produce bioactive substances called eicosanoids. Eicosanoids, such as prostaglandins, leukotrienes, and thromboxanes, have functions of smooth muscle contraction, inflammatory reaction, and platelet aggregation. The eicosanoids from each series work as autacoids and have similar functions but different potency. Eicosanoids from the $n-3$ series work mildly, showing an antagonizing effect against the strong action of eicosanoids from the n- 6 series. For details on the biochemistry and metabolism of PUFA, the readers are referred to a recent comprehensive review. ${ }^{7}$

\section{Physiological role}

Both n-6 and n-3 PUFA are important structural components of cell membranes. When incorporated into phospholipids, 
they affect cell membrane properties such as the fluidity, flexibility, permeability, and activity of membrane-bound enzymes. ${ }^{8}$ In many cases, n-3 and n- 6 series can compensate each other's function in ameliorating pathological conditions, such as growth retardation. In other situations, the biological activity of the n-3 series is more specific. Docosahexaenoic acid is incorporated into retinal cell membranes and is required for the normal development and function of the retina, suggesting its key role in vision. The phospholipids in the gray matter of the brain contain high proportions of docosahexaenoic acid and arachidonic acid, so that they are crucial to central nervous system function. ${ }^{9}$ It appears that n-3 PUFA can reduce inflammation in the brain and play a specific role in brain development and regeneration of nerve cells. ${ }^{10}$ Indeed, animal studies have shown that depletion of docosahexaenoic acid in the brain can result in learning deficits. It is unclear how docosahexaenoic acid affects brain function, but changes in docosahexaenoic acid content of neuronal cell membranes can alter the function of ion channels or membrane-associated receptors. Docosahexaenoic acid influences signaling events which are vital for neuronal survival and differentiation. ${ }^{7}$ Moreover, n- 6 and n-3 PUFA can modulate the expression of a number of genes, including those involved in fatty acid metabolism and inflammation. Although the mechanisms require further clarification, n- 6 and n-3 PUFA may regulate gene expression by interacting with specific transcription factors. ${ }^{11}$

The need for n-3 PUFA during pregnancy for fetal neurological growth and development is well recognized. The International Society for the Study of Fatty Acids and Lipids has recommended an intake of $200 \mathrm{mg}$ docosahexaenoic acid during pregnancy and lactation. ${ }^{12}$ Actually, the benefits of n-3 PUFA may well extend to the cerebral circulation, defects of which are associated with decreased cognitive ability and dementia, which are common complaints in old age. ${ }^{13}$

\section{Deficiency}

Clinical signs of essential fatty acid deficiency include a dry scaly rash, decreased growth in infants and children, increased susceptibility to infection, and poor wound healing. ${ }^{14}$ Because n-3 PUFA are highly concentrated in the brain, they are important for cognitive and behavioral function. In fact, infants who do not get enough n-3 PUFA from their mothers during pregnancy are at risk of developing vision and nerve problems. Previous studies have suggested that a dietary insufficiency of n-3 PUFA may contribute to a variety of disorders including fatigue, itchy skin, brittle hair, and nails. Other symptoms of deficiency are frequent colds, poor concentration/memory, and lack of physical endurance. Recently, researchers found that reduced levels of n-3 PUFA have deleterious consequences on synaptic function and emotional behavior, such as depression and anxiety. ${ }^{15}$

\section{$\mathrm{n}-6$ to $\mathrm{n}-3$ ratio}

Clinical studies have indicated that the ingested ratio of n- 6 to n-3 fatty acids (especially linoleic acid versus alpha-linolenic acid) is important to maintain cardiovascular health. ${ }^{16,17}$ As mentioned earlier, both n-3 and n-6 PUFA are essential and they compete for the same metabolic enzymes. Therefore, their intake ratio will significantly influence the ratio of the ensuing eicosanoids (hormones), eg, prostaglandins, leukotrienes, and thromboxanes, and will alter the metabolic function of the body. ${ }^{18}$ Metabolites of n-6 can be more inflammatory (especially arachidonic acid) than those of $n-3$. This may necessitate n-3 and n-6 PUFA being consumed in a balanced manner.

It has been observed that people nowadays tend to take excessive n- 6 fatty acids but lack n-3 fatty acids in their diet. Consequently, cells must adapt to this surplus n-6 and dietary deficiency of n-3 PUFA. Because both series regulate a number of transcription factors and interact with nuclear receptors which influence inflammatory responses and lipid metabolism, an imbalance in the $n-6: n-3$ ratio may result in altered gene regulation and expression in downstream pathways, resulting in altered protein expression and activity that can negatively affect cell membrane composition and fluidity as well as organ function. ${ }^{19}$

It has also been hypothesized that a diet rich in n-6 PUFA increases the susceptibility of low-density lipoprotein particles to oxidation, which in turn leads to atherosclerosis and cardiovascular disease. However, the detrimental effects from higher intake of n-6 PUFA have not been fully supported by evidence in humans. ${ }^{20}$ For example, a large follow-up study in the US found that the n-6:n-3 ratio was not linked to the risk of heart disease because both of them were beneficial. ${ }^{21}$

In view of the potential antagonizing effect of each eicosanoid group derived from the n- 6 and the n-3 series in the presence of conflicting scientific evidence, there have been controversies regarding the recommended adequate level of n-6 and n-3 PUFA intakes and their intake ratio. The recommended ratio of n-6:n-3 PUFA has ranged between 4 and 10 in the US and European countries, and is about 4 in Japan, based on the 2010 edition of the Dietary Reference Intakes for Japanese. ${ }^{22}$ Recent estimations of PUFA consumption in the food chain in Europe and the US showed that the ratio is still much higher than that recommended. It has been reported that healthy subjects fed with a typical Swedish diet had a n-6:n-3 
ratio measured in the serum of 4.72, whereas individuals who ate a Mediterranean-style diet resulted in a serum n-6:n-3 ratio of $2.6 .^{23}$ In general, it is difficult to resolve the issue of an optimal n-6:n-3 ratio universally because their PUFA intake is easily influenced by diet, culture, and environmental factors which vary greatly between countries. For example, the traditional Japanese diet contains 8-15 times more n-3 PUFA than does a typical non-Japanese diet. ${ }^{24}$

Instead of the n-6:n-3 ratio, another marker of cardiovascular disease risk is the n-3 index, on the basis of plasma concentrations of n-3 PUFA being a predictor of sudden cardiac death. ${ }^{25}$ This index reflects the proportion of n-3 PUFA in the membranes of red blood cells, with below 4\% indicative of coronary heart disease, whereas levels exceeding $8 \%$ give the lowest risk of cardiovascular disease events. It may thus be used to identify individuals requiring n-3 PUFA supplementation and/or dietary modification. ${ }^{26}$

\section{Clinical use for cardiovascular disease prevention}

The link between n-3 PUFA and cardiovascular disease risk reduction has been extensively studied during the past three decades. Research has shown that n-3 PUFA may prevent fatal arrhythmias (responsible for sudden cardiac death), lower blood pressure and plasma triglyceride levels, improve autonomic function, and reduce the growth rate of atherosclerotic plaque, among others, as summarized by a recent review, ${ }^{27}$ although the relative effects of docosahexaenoic acid and eicosapentaenoic acid remain uncertain. n-3 PUFA supplementation is often used in the secondary prevention of fatal and nonfatal myocardial infarction. ${ }^{28}$ Its therapeutic actions have been recently extended to the prevention and treatment of heart failure. A large prospective cohort study of nearly 60,000 Japanese found an inverse association between fish and n-3 PUFA consumption and cardiovascular disease mortality, especially for heart failure. ${ }^{29}$ The finding raised the possibility that n-3 PUFA supplementation and pharmacological doses may be needed in different stages of heart failure.

In relation to clinical use, evidence from three systematic reviews supports a neutral effect on cardiovascular disease mortality for n-3 PUFA intake of 400-7000 mg per day, ${ }^{30-32}$ whereas no scientific data were available beyond $7000 \mathrm{mg} /$ day. Other systematic reviews focused on surrogate markers of cardiovascular disease such as lipid profiles, the majority of which found a protective effect of n-3 PUFA on triglycerides and a neutral or small adverse effect on total cholesterol, low density lipoprotein cholesterol, and high density lipoprotein cholesterol. ${ }^{33-39}$ Moreover, there is little evidence on n-3 PUFA sourced from vegetables in relation to cardiovascular disease. Although a wide range of adult populations, predominantly from America and Europe, were considered in these reviews, it should be cautioned that some of the systematic reviews included subjects who had already experienced a cardiovascular disease event and were secondary prevention trials. As such, the results must be interpreted with caution because these studies could not be removed from the systematic review or meta-analysis. In addition to different study designs and populations, the variability in results across clinical trials may be due to confounding factors, such as the patients' baseline diet, types and doses of eicosapentaenoic acid and docosahexaenoic acid given, duration of treatment, and patient compliance. ${ }^{6}$

The mechanisms by which n-3 PUFA reduces cardiovascular disease morbidity and mortality are manifold. ${ }^{40}$ The activities have been extensively investigated in human intervention trials and in vitro mechanistic studies. A detailed discussion of the potential mechanisms is beyond the scope of this paper. There appear to be two plausible mechanisms. ${ }^{41}$ Firstly, the suppression of cardiac arrhythmias, ${ }^{41}$ by a) directly inhibiting voltage-gated sodium channels and L-type calcium channels, resulting in a longer relative refractory period and in an increased voltage required for membrane depolarization, which in turn reduces heart rate; b) improves left ventricular efficiency, and reduces blood pressure, which indirectly contribute to lowering the heart rate. Secondly, n-3 PUFA can reduce atherogenesis, occurring through the modulation of specific atherothrombotic risk factors, decreased platelet aggregation, reduced plasma triglycerides and blood pressure, and above all, the regulation of systemic and local inflammation underlying plaque inception, progression, and instability. ${ }^{27,41}$ For patients with heart failure, n-3 PUFA may also invoke stimulation of peroxisome proliferator-activated receptor alpha receptors, improve mitochondrial function, and downregulate the anti-inflammatory pathway. ${ }^{42}$ The readers are referred to recent reviews for more details. ${ }^{19,41}$

It should be remarked that the suggested antiarrhythmic effect of n-3 PUFA remains a controversial issue. Clinical studies on ventricular arrhythmias have reported mixed results, ${ }^{43}$ while a recent clinical trial on n-3 PUFA supplementation concluded no reduction in risk of atrial fibrillation after coronary artery bypass graft surgery. ${ }^{44}$

\section{Recommended intake and side effects}

Several guidelines have been issued by scientific bodies. For example, the American Heart Association had released a 
scientific statement on the effects of n-3 PUFA on heart function (including antiarrhythmic effects), hemodynamics (cardiac mechanics), and arterial endothelial function. ${ }^{45}$ For patients with documented coronary heart disease, the American Heart Association currently endorses the use of n-3 PUFA at a dose of approximately $1000 \mathrm{mg}$ /day of combined docosahexaenoic acid and eicosapentaenoic acid, either in the form of fatty fish or fish oil supplements (in capsules or liquid form). For patients who need to lower triglycerides, 2000-4000 mg should be taken as capsules under a physician's care. The American Heart Association has further recommended everyone to "eat a variety of (preferably oily) fish at least twice a week. Include oils and foods rich in alpha-linolenic acid (flaxseed, canola, and soybean oils; flaxseed and walnuts)" ${ }^{45}$

The National Heart Foundation of Australia similarly recommends the consumption of $500 \mathrm{mg}$ of combined docosahexaenoic acid and eicosapentaenoic acid every day through a combination of two to three $150 \mathrm{~g}$ serves of oily fish per week, fish oil supplements (capsules or liquid), and food and drinks enriched with marine n-3 PUFA, in addition to at least $2 \mathrm{~g}$ of alpha-linolenic acid intake per day. ${ }^{46}$ However, the Australian National and Medical Research Council estimated that consuming at least $90 \mathrm{mg} /$ day for women and $160 \mathrm{mg} /$ day for men of n-3 PUFA (marine source) and $800 \mathrm{mg} /$ day for women and $1300 \mathrm{mg} /$ day for men of alpha-linolenic acid should be adequate for good health. To prevent chronic disease, target intake levels for combined docosahexaenoic acid, eicosapentaenoic acid, and docosapentaenoic acid are $430 \mathrm{mg} /$ day for women and $610 \mathrm{mg} / \mathrm{day}$ for men, with the upper limit being set at $3000 \mathrm{mg} /$ day. ${ }^{47} \mathrm{In}$ addition to over the counter supplements (fish oils), a standardized encapsulated concentrate of eicosapentaenoic acid and docosahexaenoic acid as a prescription drug is readily available in the market.

The International Society for the Study of Fatty Acids and Lipids recommends an intake of alpha-linolenic acid of $0.7 \%$ of energy, and a minimum intake of $500 \mathrm{mg}$ /day of combined eicosapentaenoic acid and docosahexaenoic acid. ${ }^{12}$ In spite of the global consensus on the $500 \mathrm{mg}$ intake level for healthy adults set by various government organizations and professional bodies, and a separate recommendation of $200 \mathrm{mg}$ docosahexaenoic acid for pregnant and lactating women, ${ }^{12}$ with the exception of Japan and a few isolated places, most people in the world are not consuming sufficient n-3 PUFA for optimal health. For example, the average n-3 PUFA intake of Australians has been estimated at $246 \mathrm{mg}$ /day, well below the Japanese median intake of $810 \mathrm{mg} /$ day in $2003 .^{5}$

Table 1 summarizes the recommendations from various guidelines. It is noteworthy that docosahexaenoic acid
Table I Summary of recommended n-3 PUFA intake

\begin{tabular}{|c|c|c|c|}
\hline $\begin{array}{l}\text { Population } \\
\text { group }\end{array}$ & $\begin{array}{l}\text { Recommended } \\
\text { daily intake }\end{array}$ & n-3 PUFA & Source \\
\hline Healthy adults & $\begin{array}{l}500 \mathrm{mg} \\
2000 \mathrm{mg}\end{array}$ & $\begin{array}{l}\text { DHA + EPA } \\
\text { ALA }\end{array}$ & $\begin{array}{l}\text { Fish/supplement } \\
\text { Oils and foods }\end{array}$ \\
\hline $\begin{array}{l}\text { Pregnant and } \\
\text { lactating women }\end{array}$ & $200 \mathrm{mg}$ & $\mathrm{DHA}$ & Supplement \\
\hline $\begin{array}{l}\text { Patients with } \\
\text { CHD }\end{array}$ & $1000 \mathrm{mg}$ & $D H A+E P A$ & Fish/supplement \\
\hline $\begin{array}{l}\text { Patients to lower } \\
\text { triglycerides }\end{array}$ & $\begin{array}{l}2000-4000 \mathrm{mg} \\
\text { under physician's } \\
\text { care }\end{array}$ & $D H A+E P A$ & $\begin{array}{l}\text { Capsule } \\
\text { supplement }\end{array}$ \\
\hline
\end{tabular}

Abbreviations: CHD, coronary heart disease; PUFA, polyunsaturated fatty acids; ALA, alpha-linolenic acid; DHA, docosahexaenoic acid; EPA, eicosapentaenoic acid.

and eicosapentaenoic acid differ in their ability to promote various effects of n-3 PUFA. Indeed, the overlapping and separate mechanisms by which docosahexaenoic acid and eicosapentaenoic acid prevent and reverse cardiovascular disease are still unclear. ${ }^{19}$ The optimal doses and ratios of docosahexaenoic acid to eicosapentaenoic acid are difficult to determine, as both are present in most fish especially oily fish, generally in a $2: 1$ ratio. $^{48}$

As far as safety is concerned, unlike cardiac pharmaceuticals, n-3 PUFA supplementations are well tolerated. High doses of n-3 PUFA may in theory increase the bleeding time by inhibiting the arachidonic acid pathway. Clinically, it does not pose a significantly increased risk of bleeding for patients undergoing coronary artery bypass grafting, carotid endarterectomy, or femoral artery catheterization, even at high doses combined with antiplatelet therapy or warfarin, ${ }^{27}$ though it may have adverse effects in patients with angina. ${ }^{49}$ The most commonly observed side effects are nausea, gastrointestinal symptoms, fish-scented halitosis, and dysgeusia, which are not considered as major health hazards. ${ }^{42}$ There is also some concern about ingestion of mercury when fishes are eaten, particularly for species like shark and swordfish. Nevertheless, commonly consumed oily fish such as salmon, skipjack tuna, mackerel, trout, and sardines contain very low levels of mercury, while purified fish oils in pharmaceutical grade capsules typically have negligible amounts. ${ }^{50}$

\section{Summary and perspectives}

Cardiovascular disease will continue to be the leading cause of mortality worldwide due to escalating aging populations coupled with an increasing incidence of obesity and type 2 diabetes, which share many of the emerging risk factors for cardiovascular disease.

The n-3 PUFA series plays a crucial role in brain function, as well as normal growth and development. Fish, particularly 
fatty fish like salmon, mackerel, herring, lake trout, sardines, and skipjack tuna, is a good source of n-3 PUFA. The American Heart Association dietary guidelines currently recommend eating fish at least two times (two servings) a week. Each serving is 3.5 ounces cooked, or about three quarters of a cup of flaked fish. ${ }^{51}$ This amount is equivalent to about $500 \mathrm{mg} /$ day of combined eicosapentaenoic acid and docosahexaenoic acid intake for individuals without underlying overt cardiovascular disease. ${ }^{27}$

Given the low prevalence of cardiovascular disease in populations such as the Japanese whose diet is largely based on fish, dietary modification towards greater fish consumption is desirable. However, when adherence to the recommended diet regimen is inadequate or availability of the recommended fish species is limited, it might be necessary to administer purified supplements. ${ }^{13}$

An ongoing debate concerns the $n-6: n-3$ ratio. Based on in vitro competition mechanisms, reducing the ratio should in theory lessen the incidence of degenerative diseases, including those with an inflammatory component. However, the validity of this hypothesis in vivo has been questioned. ${ }^{17}$ Both n-3 and n-6 PUFA are essential. From a nutritional viewpoint, the absolute ratio might not be a useful concept because at any ratio, both intakes could be deficient. ${ }^{40}$ It might be better to consider PUFA individually rather than as a ratio (which does not distinguish between alpha-linolenic acid and eicosapentaenoic acid + docosahexaenoic acid) and to provide evidencebased dietary advice to increase n-3 PUFA intake rather than just decreasing n-6 PUFA. Assessment of individual needs may be based on the $n-3$ red blood cell index. ${ }^{26}$ However, the index may not be a useful prognostic risk marker following an acute coronary syndrome, ${ }^{52}$ so further studies are needed to validate this biomarker for cardiovascular risk.

In spite of the pitfalls of meta-analyses, epidemiological studies have suggested the potential benefits of n-3 PUFA on cardiovascular health. Randomized controlled intervention trials continue to show the success of n-3 PUFA in primary and secondary cardiovascular disease prevention, while evidence from cellular and molecular research indicates that the cardioprotective effects result from a synergism between multiple intricate mechanisms that involve anti-inflammatory, antiatherosclerotic, and antiarrhythmic effects.

Although considerable research ranging from in vitro to animal to human studies has been dedicated to elucidating the activities of n-3 PUFA on the cardiovascular system, for practical and financial reasons, most investigations did not measure PUFA levels in plasma or in red blood cells before and after intervention. This limits the identification of dose-dependent correlations between plasma PUFA variations (a reliable marker of intake) and clinical outcomes. ${ }^{40}$

Generally, n-3 PUFA supplementation has few side effects and is recognized as safe by the US Food and Drug Administration. The target consumption range appears to be $1000 \mathrm{mg} /$ day for patients with coronary heart disease or heart failure, but further clinical studies are needed to determine optimal dosing for various populations and the relative ratio of docosahexaenoic acid to eicosapentaenoic acid that can provide maximum protection for those at risk of cardiovascular disease and in the treatment of cardiovascular diseaserelated disorders. High quality, randomized, controlled trials are also needed to clarify the benefits of alpha-linolenic acid for cardiovascular health. It is unclear whether alpha-linolenic acid prevents recurrent coronary events in view of the few studies available. Regardless of the scientific evidence, for people who still wish to supplement their diet with fish oil capsules, the advantage is the virtual lack of any deleterious and side effects of n-3 PUFA and the possibility to find eicosapentaenoic acid/docosahexaenoic acid as oral preparations without prescription at a relatively low cost.

In conclusion, more research is required to clarify the role of n-3 PUFA supplementation. Recent clinical trials found that n-3 PUFA supplementation had little prognostic effect for patients receiving good medical treatment after myocardial infarction..$^{53,54}$ The dose-dependent benefits should be investigated among patients receiving optimal medical therapy for cardiovascular protection, including background use of statins, ACE inhibitors, beta-blockers, and aspirin. ${ }^{42}$ Further research is also warranted to provide recommendations for other patient groups (eg, diabetes ${ }^{55}$ ). Nevertheless, given the public health focus on the epidemic of cardiovascular disease, there is certainly scope for the inclusion of n-3 PUFA therapy as part of a comprehensive cardiovascular disease management package. It should also be noted that the diverse biological roles played by n-3 PUFA extend beyond the cardiovascular system. It is envisaged that further evidence-based recommendations will be issued in the near future.

\section{Disclosure}

The authors report no conflicts of interest in this work.

\section{References}

1. World Health Organization. Cardiovascular diseases (CVDs). Available from: http://www.who.int/mediacentre/factsheets/fs317/en/index.html. Accessed September 1, 2011.

2. Centers for Disease Control and Prevention. Heart Disease and Stroke Prevention. Addressing the nation's leading killers: at a glance 2010. Available from: http://www.cdc.gov/chronicdisease/resources/ publications/AAG/dhdsp.htm. Accessed September 1, 2011. 
3. National Heart Foundation of Australia. Data and statistics. Available from: http:/www.heartfoundation.org.au/information-for-professionals/ data-and-statistics. Accessed September 1, 2011.

4. Mozaffarian D. JELIS, fish oil and cardiac events. Lancet. 2007; 369(9567):1062-1063.

5. Meyer BJ. Are we consuming enough long chain omega-3 polyunsaturated fatty acids for optimal health? Prostaglandins Leukot Essent Fatty Acids. May 13, 2011. [Epub ahead of print.]

6. Chan EJ, Cho L. What can we expect from omega-3 fatty acids? Cleve Clin J Med. 2009;76(4):245-251.

7. Russo GL. Dietary n-6 and n-3 polyunsaturated fatty acids: from biochemistry to clinical implications in cardiovascular prevention. Biochem Pharmacol. 2009;77(6):937-946.

8. Stillwell W, Wassall SR. Docosahexaenoic acid: membrane properties of a unique fatty acid. Chem Phys Lipids. 2003;126(1):1-27.

9. Innis SM. Perinatal biochemistry and physiology of long-chain polyunsaturated fatty acids. J Pediatr. 2003;143(4 Suppl):S1-S8.

10. Kalmijin S. Fatty acid intake and the risk of dementia and cognitive decline: a review of clinical and epidemiological studies. J Nutr Health Aging. 2000;4(4):202-207.

11. Sampath H, Ntambi JM. Polyunsaturated fatty acid regulation of gene expression. Nutr Rev. 2004;62(9):333-339.

12. International Society for the Study of Fatty Acids and Lipids. Recommendations for intake of polyunsaturated fatty acids in healthy adults Available from: http://www.issfal.org. Accessed September 1, 2011.

13. Calzolari I, Fumagalli S, Marchionni N, Di Bari M. Polyunsaturated fatty acids and cardiovascular disease. Curr Pharm Des. 2009;15(36): 4094-4102.

14. Jeppesen PB, Hoy CE, Mortensen PB. Essential fatty acid deficiency in patients receiving home parenteral nutrition. Am J Clin Nutr. 1998;68(1):126-133.

15. Lafourcade M, Larrieu T, Mato S, et al. Nutritional omega-3 deficiency abolishes endocannabinoid-mediated neuronal functions. Nat Neurosci. 2011;14(3):345-350.

16. Okuyama H. High n-6 to n-3 ratio of dietary fatty acids rather than serum cholesterol as a major risk factor for coronary heart disease. Eur J Lipid Sci Technol. 2001;103(6):418-422.

17. Griffin BA. How relevant is the ratio of dietary n-6 to n-3 polyunsaturated fatty acids to cardiovascular disease risk? Evidence from the OPTILIP study. Curr Opin Lipidol. 2008;19(1):57-62.

18. Tribole EF, Thompson RL, Harrison RA, et al. Excess omega-6 fats thwart health benefits from omega-3 fats. BMJ. 2006;332(7544):752-760.

19. Adkins Y, Kelley DS. Mechanisms underlying the cardioprotective effects of omega-3 polyunsaturated fatty acids. J Nutr Biochem. 2010;21(9):781-792.

20. Willett WC. The role of dietary $n-6$ fatty acids in the prevention of cardiovascular disease. J Cardiovasc Med (Hagerstown). 2007;8 Suppl 1:S42-S45.

21. Mozaffarian D, Ascherio A, Hu FB, et al. Interplay between different polyunsaturated fatty acids and risk of coronary heart disease in men. Circulation. 2005;111(2):157-164.

22. Japanese Ministry of Health, Labour and Welfare. Dietary Reference Intakes for Japanese (2010 edition). Available from: http://www.mhlw go.jp/bunya/kenkou/sessyu-kijun.html. Accessed September 1, 2011.

23. Ambring A, Johansson M, Axeisen M, Gan L, Strandvik B, Friberg P. Mediterranean-inspired diet lowers the ratio of serum phospholipid n-6 to n-3 fatty acids, the number of leukocytes and platelets, and vascular endothelial growth factor in healthy subjects. Am J Clin Nutr. 2006;83(3):575-581.

24. Iso $H$, Kobayashi $M$, Ishihara $J$, et al. Intake of fish and $n 3$ fatty acids and risk of coronary heart disease among Japanese: the Japan Public Health Center-Based (JPHC) Study Cohort I. Circulation. 2006;113(2):195-202.

25. Harris WS. The omega-3 index as a risk factor for coronary heart disease. Am J Clin Nutr. 2008;87(6):1997S-2002S.

26. Harris WS. The omega-3 index: from biomarker to risk marker to risk factor. Curr Atheroscler Rep. 2009;11(6):411-417.
27. Lavie CJ, Milani RV, Mehra MR, Ventura HO. Omega-3 polyunsaturated fatty acids and cardiovascular diseases. J Am Coll Cardiol. 2009; 54(7):585-594.

28. Patel JV, Tracey I, Hughes EA, Lip GY. Omega-3 polyunsaturated fatty acids: a necessity for a comprehensive secondary prevention strategy. Vasc Health Risk Manag. 2009;5:801-810.

29. Yamagishi K, Iso H, Date $\mathrm{C}$, et al. Fish, omega-3 polyunsaturated fatty acids, and mortality from cardiovascular diseases in a nationwide community-based cohort of Japanese men and women the JACC (Japan Collaborative Cohort Study for Evaluation of Cancer Risk) Study. J Am Coll Cardiol. 2008;52(12):988-996.

30. Hooper L, Thompson RL, Harrison RA, et al. Omega 3 fatty acids for prevention and treatment of cardiovascular disease. Cochrane Database Syst Rev. 2004;18(4):CD003177.

31. Studer M, Briel M, Leimenstoll B, Glass TR, Bucher HC. Effect of different antilipidemic agents and diets on mortality: a systematic review. Arch Intern Med. 2005;165(7):725-730.

32. Wang C, Harris WS, Chung M, et al. n-3 Fatty acids from fish or fish-oil supplements, but not alpha-linolenic acid, benefit cardiovascular disease outcomes in primary- and secondary-prevention studies: a systematic review. Am J Clin Nutr. 2006;84(1):5-17.

33. Balk EM, Lichtenstein AH, Chung M, Kupelnick B, Chew P, Lau J. Effects of omega-3 fatty acids on coronary restenosis, intima-media thickness, and exercise tolerance: A systematic review. Atherosclerosis. 2006;184(2):237-246.

34. Balk EM, Lichtenstein AH, Chung M, Kupelnick B, Chew P, Lau J. Effects of omega-3 fatty acids on serum markers of cardiovascular disease risk: a systematic review. Atherosclerosis. 2006;189(1): 19-30.

35. Castro IA, Barroso LP, Sinnecker P. Functional foods for coronary heart disease risk reduction: a meta-analysis using a multivariate approach. Am J Clin Nutr. 2005;82(1):32-40.

36. Eslick GD, Howe PRC, Smith C, Priest R, Bensoussan A. Benefits of fish oil supplementation in hyperlipidemia: a systematic review and meta-analysis. Int J Cardiol. 2009;136(1):4-16.

37. Lee KW, Lip GY. Effects of lifestyle on hemostasis, fibrinolysis, and platelet reactivity: a systematic review. Arch Intern Med. 2003;163(19): 2368-2392.

38. Mensink RP, Zock PL, Kester AD, Katan MB. Effects of dietary fatty acids and carbohydrates on the ratio of serum total to HDL cholesterol and on serum lipids and apolipoproteins: a meta-analysis of 60 controlled trials. Am J Clin Nutr. 2003;77(5):1146-1155.

39. Wendland E, Farmer A, Glasziou P, Neil A. Effect of alpha linolenic acid on cardiovascular risk markers: a systematic review. Heart 2006;92(2):166-169.

40. Richard D, Bausero P, Schneider C, Visioli F. Polyunsaturated fatty acids and cardiovascular disease. Cell Mol Life Sci. 2009;66(20): 3277-3288.

41. Massaro M, Scoditti E, Carluccio MA, De Caterina R. Basic mechanisms behind the effects of n-3 fatty acids on cardiovascular disease. Prostaglandins Leukot Essent Fatty Acids. 2008;79(3-5): 109-115.

42. Watts GF, Mori TA. Recent advances in understanding the role and use of marine $\omega 3$ polyunsaturated fatty acids in cardiovascular protection. Curr Opin Lipidol. 2011;22(1):70-71.

43. Jenkins DJ, Josse AR, Dorian P, et al. Heterogeneity in randomized controlled trials of long chain (fish) omega-3 fatty acids in restenosis, secondary prevention and ventricular arrhythmias. J Am Coll Nutr. 2008;27(3):367-378.

44. Saravanan P, Bridgewater B, West AL, O'Neill SC, Calder PC, Davidson NC. Omega-3 fatty acid supplementation does not reduce risk of atrial fibrillation after coronary artery bypass surgery: a randomized, double-blind, placebo-controlled clinical trial. Circ Arrhythm Electrophysiol. 2010;3(1):46-53.

45. Kris-Etherton PM, Harris WS, Appel LJ; for American Heart Association Nutrition Committee. Fish consumption, fish oil, omega-3 fatty acids, and cardiovascular disease. Circulation. 2002;106(21):2747-2757. 
46. National Heart Foundation of Australia. Position statement: Fish, fish oils, n-3 polyunsaturated fatty acids and cardiovascular health. Updated November 2008. Available from: www.heartfoundation.org. au. Accessed September 1, 2011.

47. National Health and Medical Research Council. Nutrient Reference Values for Australia and New Zealand including Recommended Dietary Intakes. Canberra, ACT: Australian Government Department of Health and Ageing; 2006.

48. USDA Agricultural Research Service. Nutrient Data Laboratory. Available from: http://www.ars.usda.gov/nutrientdata. Accessed September 1, 2011.

49. Zhao YT, Chen Q, Sun YX, et al. Prevention of sudden cardiac death with omega-3 fatty acids in patients with coronary heart disease: a meta-analysis of randomized controlled trials. Ann Med. 2009;41(4): 301-310.

50. Saravanan P, Davidson NC, Schmidt EB, Calder PC. Cardiovascular effects of marine omega-3 fatty acids. Lancet. 2010;376(9740): $540-550$.
51. American Heart Association. Fish and omega-3 fatty acids. Available from: http://www.amhrt.org/HEARTORG/GettingHealthy/ NutritionCenter/HealthyDietGoals/Fish-and-Omega-3-Fatty-Acids_ UCM_303248_Article.jsp. Accessed September 1, 2011.

52. Aarsetoey H, Pönitz V, Grundt H, Staines H, Harris WS, Nilsen DWT. (n-3) fatty acid content of red blood cells does not predict risk of future cardiovascular events following an acute coronary syndrome. J Nutr. 2009;139(3):507-513.

53. Kromhout D, Giltay EJ, Geleijnse JM; Alpha Omega Trial Group. n-3 fatty acids and cardiovascular events after myocardial infarction. $N$ Engl J Med. 2010;363(21):2015-2026.

54. Rauch B, Schiele R, Schneider S, et al. OMEGA, a randomized, placebocontrolled trial to test the effect of highly purified omega-3 fatty acids on top of modern guideline-adjusted therapy after myocardial infarction. Circulation. 2010;122(21):2152-2159.

55. McEwen B, Morel-Kopp MC, Tofler G, Ward C. Effect of omega-3 fish oil on cardiovascular risk in diabetes. Diabetes Educ. 2010;36(4): 565-584.
Nutrition and Dietary Supplements

\section{Publish your work in this journal}

Nutrition and Dietary Supplements is an international, peer-reviewed, open access journal focusing on research into nutritional requirements in health and disease, impact on metabolism and the identification and optimal use of dietary strategies and supplements necessary for normal growth and development. The journal welcomes papers covering

\section{Dovepress}

original research, basic science, clinical \& epidemiological studies, reviews and evaluations, guidelines, expert opinion and commentary, case reports and extended reports. The manuscript management system is completely online and includes a very quick and fair peer-review system, which is all easy to use.

Submit your manuscript here: http://www.dovepress.com/nutrition-and-dietary-supplements-journal 